\title{
Disorder-induced Floquet Topological Insulators in Photonic Systems
}

\author{
Paraj T. Bhattacharjee $^{1}$, Netanel H. Lindner ${ }^{2}$, Mikael C. Rechtsman ${ }^{2}$, Gil Refael ${ }^{1}$ \\ ${ }^{1}$ Department of Physics, California Institute of Technology, Pasadena, California 91125, USA \\ ${ }^{2}$ Department of Physics, Technion - Israel Institute of Technology, Haifa 32000, Isarel \\ refael@caltech.edu
}

\begin{abstract}
We propose the photonic topological Anderson insulator (FTAI), the first realization of the FTAI phase in any physical context (including condensed matter and cold atoms). In this phase, disorder counterintuitively induces a topological transition that breaks Anderson localization and leads to robust transport.
\end{abstract}

Ocis codes: $230.7370,240.0240,350.4238$

Topological insulators are materials that insulate in their bulk, but conduct electricity on their surfaces [1-3]. In two dimensions, they possess states that conduct electricity on their edges and cannot be scattered (either backwards along the edge or into the bulk) by any form of imperfection or disorder - this is one aspect of "topological protection". This mechanism gives rise to striking robustness that has allowed for the study of collective quantum phenomena such as the integer, fractional, and spin quantum Hall effects. Recently, an ongoing theoretical effort to realize topological insulation and protection in optics culminated in the first experimental observation of a photonic topological insulators (PTI) [4] in optics, as demonstrated in an array of evanescently coupled helical waveguides. The question of disorder lies at the heart of topological protection, since in the non-scattering edge states can act to counter the conventional mechanism of Anderson localization. While in two-dimensional non-topological systems, Anderson localization acts to exponentially localize all eigenstates, this is not the case in topological systems. Topological edge states act against Anderson localization, and can lead to delocalized eigenstates that span the extent of the system.

Here, we propose a "photonic topological Anderson insulator:" a disordered PTI that exhibits disorder-induced transport. We show that under certain conditions, the introduction of disorder to a Floquet PTI (mentioned above [4]) leads to the closing of a non-topological band gap and reopening of a topological band gap. This in turn leads to the presence of a photon-current-carrying topological edge state, which is extended throughout the system despite the presence of disorder. This would be the first realization - in any form, including condensed matter or cold atoms systems - of a topological Anderson insulator [5], predicted several years ago to arise in some topological systems. We expect that this phase can be realized in photonic lattices, for example with femtosecond-laser-written waveguide arrays [6].

The mechanism in which disorder induces a topological phase may be described as follows. Given a helical waveguide array in a honeycomb pattern (shown in Fig. 1(a) with the A sublattice in red and the B in blue) with helicity radius $R$ and spatial frequency $\Omega$, the diffraction of light through the structure is described by the paraxial Schrodinger equation:

$$
i \partial_{z} \psi_{n}=t \sum_{\langle m\rangle} e^{-i \mathbf{A}(\mathbf{z}) \cdot \mathbf{r}_{m n}} \psi_{m}+M v_{n} \psi_{n}+w_{n} \psi_{n}
$$

where $z$ is the distance in the light propagation direction; ${ }_{n}$ represents the optical amplitude on the $n^{\text {th }}$ waveguide; the summation is taken over nearest neighbors; $\boldsymbol{A}=A_{0}(\cos \Omega z, \sin \Omega z) ; A_{0}=k_{0} \Omega R$ is the strength of the vector potential; $k_{0}$ is the ambient wavenumber in the medium; $v_{n}$ is a staggered potential that takes on the value 1 on sublattice $A$ and -1 on sublattice $B$ of the honeycomb structure; $M$ is the strength of the sublattice potential; and $w_{n}$ represents the random on-site disorder. In the absence of helical rotation, $A_{0}=0$, and the presence of the staggered potential (of strength $M$ ) will open up a 
topologically trivial band gap (shown in Fig. 1(b)). Conversely, for $M=0$, the presence of helical rotation acts to open a topological gap (shown in Fig. 1(c)). Consider the case where both $A_{0}$ and $M$ are non-zero, but where $M$ is sufficiently large to open a non-topological band gap. In this scenario, we have shown using the self-consistent Born approximation that under the influence of on-site disorder, $\mathrm{M}$ is effectively reduced on average. This allows the strength of the helicity to outweigh that of the staggered potential and for a topological band gap to open. This, in turn, results in an extended edge state in the system. In Fig 1(d), we show a phase diagram where the topological invariant (the 'Bott index') is plotted against the propagation constant (also called the quasi-energy), $\beta$, and the disorder strength, $\left\langle w_{n}\right\rangle$. It is clear that the non-trivial phase is induced as a result of non-zero disorder. Figure 1 (e) shows the wavefunction at fixed when the initial excitation is at the left edge, center, and right edge of the array, respectively. The presence of the topological edge state in the first and last cases is evident.
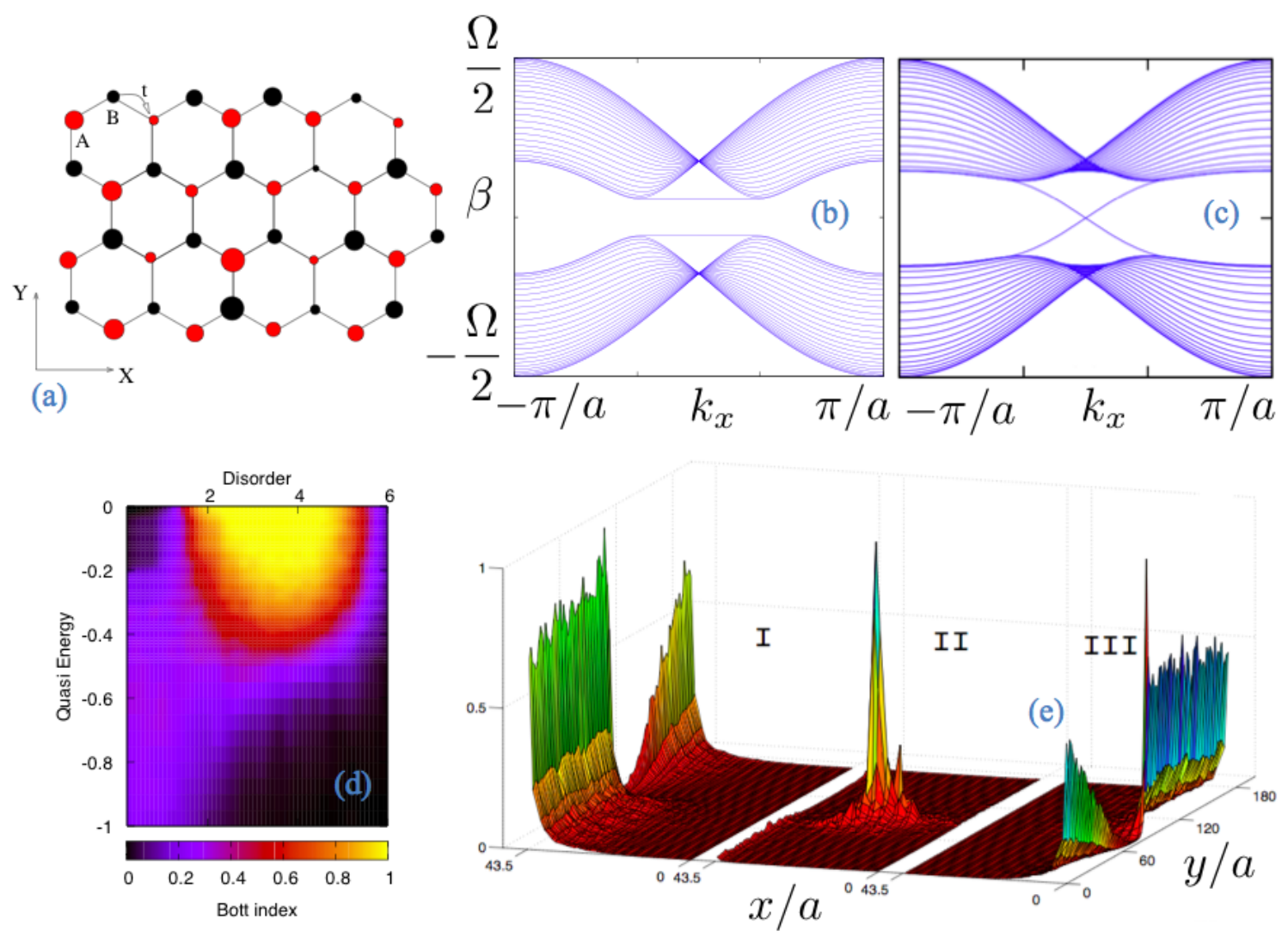

Figure 1. (a): Schematic of honeycomb waveguide array with random on-site potential; (b), (c): Band structures for the topologically trivial $\left(A_{0}=0\right)$ and non-trival phases $(M=0)$, respectively; (d): Phase diagram of the Bott index in disorder-energy space; (e) Edge states in I and III with bulk excitations on left and right edges; bulk state in II results from a bulk excitation.

\section{$\underline{\text { References }}$}

[1] C. L. Kane and E. J. Mele, Phys. Rev. Lett. 95, 226801 (2005).

[2] B. A. Bernevig, T. L. Hughes, and S.-C. Zhang, Science 314, 1757 (2006).

[3] M. Z. Hasan and C. L. Kane, Rev. Mod. Phys. 82, 3045 (2010).

[4] M. C. Rechtsman, J. M. Zeuner, Y. Plotnik, Y. Lumer, D. Podolsky, F. Dreisow, S. Nolte, M. Segev, and A. Szameit, Nature 496, 196 (2013).

[5] J. Li, R.-L. Chu, J. K. Jain, and S.-Q. Shen, Phys. Rev. Lett. 102, 136806 (2009).

[6] A. Szameit and S. Nolte, J. Phys. B At. Mol. Opt. Phys. 43, 163001 (2010). 